Check for updates

Cite this: Chem. Commun., 2020 56, 14423

Received 2nd September 2020, Accepted 26th October 2020

DOI: $10.1039 / \mathrm{d} 0 \mathrm{cc} 05893 \mathrm{a}$

rsc.li/chemcomm

\section{Visualizing antithrombin-binding 3-O-sulfated heparan sulfate motifs on cell surfaces $\dagger$}

\author{
Mausam Kalita, (D) a Jie Shi Chua, ${ }^{a}$ Rio S. Boothello, (D) ${ }^{b c}$ April Joice, ${ }^{a}$ \\ Orlando Antelope, ${ }^{a}$ Anindita Roy, ${ }^{a}$ Pon Velayutham Anandh Babu, id d \\ Yukio Saijoh, (D) ${ }^{\mathrm{e}}$ Umesh R. Desai (D) ${ }^{\mathrm{bc}}$ and Balagurunathan Kuberan (D) *a
}

To map the cellular topography of the rare 3-O-sulfated structural motif of heparan sulfate (HS), we constructed quantum dot-based probes for antithrombin and FGF2, which reveal widely different distribution of the targeted HS motifs. The technology helps show that old and young aortic endothelia display widely different levels of the antithrombin-binding 3-O-sulfated HS motif.

Heparan sulfate proteoglycans (HSPGs), such as syndecans and glypicans, are present on virtually all mammalian cell surfaces and modulate a number of processes such as growth, differentiation and morphogenesis. ${ }^{1}$ The glycosaminoglycan (GAG) component of HSPGs is heparan sulfate (HS), a polysaccharide of alternating glucosamine and uronic acid residues $\neq$ that are not uniformly sulfated resulting in a diverse array of structural and conformational microdomains within the same chain. The diversity of microdomains results in a diversity of biological functions, owing to direct engagement of proteins such as plasma proteins, extracellular matrix (ECM) proteins and cell surface receptors. Classic examples of this include the HS-antithrombin (AT) ${ }^{2-4}$ and HSfibroblast growth factor $2(\mathrm{FGF} 2)^{5-8}$ systems that have yielded key insights into recognition and regulation by GAGs. Human plasma AT binds tightly to a rare 3 -O-sulfated pentasaccharide motif in $\mathrm{HS},{ }^{3,4}$ whereas FGF2 is known to bind to common tetrasaccharide motifs in $\mathrm{HS}^{6-8}$ with good affinity (Fig. 1).

The rare 3-O-sulfate (3-OS) group of $\mathrm{HS}$ is introduced by one of the six human 3-O-sulfotransferases (3OSTs), which possess

\footnotetext{
${ }^{a}$ Departments of Medicinal Chemistry and Bioengineering, University of Utah,

Salt Lake City, UT 84112, USA. E-mail: kuby@pharm.utah.edu

${ }^{b}$ Department of Medicinal Chemistry, School of Pharmacy,

Virginia Commonwealth University, Richmond, VA 23298, USA.

E-mail: urdesai@vcu.edu

${ }^{c}$ Institute for Structural Biology, Drug Discovery and Development,

Virginia Commonwealth University, Richmond, VA 23219, USA

${ }^{d}$ Department of Nutrition and Integrative Physiology, University of Utah,

Salt Lake City, UT 84112, USA

${ }^{e}$ Department of Neurobiology and Anatomy, University of Utah School of Medicine, Salt Lake City, UT 84112, USA

$\dagger$ Electronic supplementary information (ESI) available. See DOI: 10.1039/ d0cc05893a
}

differing substrate specificities, thereby generating different 3-Osulfated microdomains in $\mathrm{HS}^{3,9}$ Interestingly, 3-O-sulfation of HS chains is tissue-type dependent, e.g., endothelial cells have approximately one 3-OS group per 100 HS disaccharides, whereas follicular fluid has nearly six and Reichert's basement membrane contains five to ten 3-OS groups. ${ }^{10-12}$ Yet, are these 3-OS groups uniformly distributed or are they present in select locales in vivo remains unknown. Considering that growing evidence suggests spatial and temporal regulation of enzymes involved in HS biosynthesis, ${ }^{13,14}$ it is critical to develop readily applicable tools to help elucidate topography of HS, especially for rare structural motifs.

Although several probes for spatio-temporal visualization of DNA, mRNA and proteins have been developed, ${ }^{15-17}$ probes for monitoring spatiotemporal changes in GAGs remain limited. Few examples known in the literature relate to tracking proteins, such as HIT-1 Tat or HSV gB, surfing over HSPGs ${ }^{18,19}$ or tracking common HS motifs through the use of antibodies. ${ }^{20}$ Likewise, a radioactive probe $\left({ }^{125} \mathrm{I}\right.$-AT) has also been explored for imaging HS too with limited applicability. ${ }^{21}$ Most importantly, none of these probes offer insight into spatio-temporal topography of rare and specific HS sequences. This is critical for elucidating fundamental biochemical principles that regulate HS biosynthesis as well as for deciphering spatio-temporal
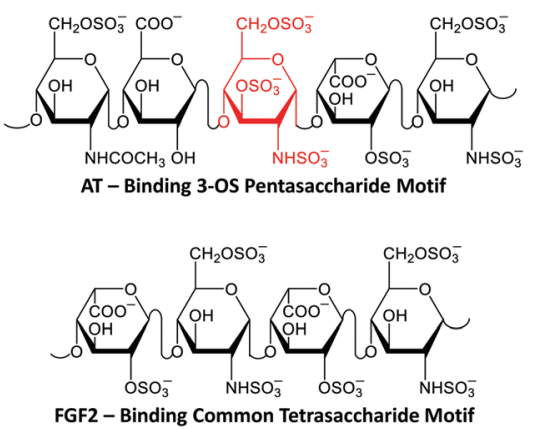

Fig. 1 Structures of the rare 3-O-sulfated motif binding to antithrombin and the common tetrasaccharide motif binding to FGF2. 
A

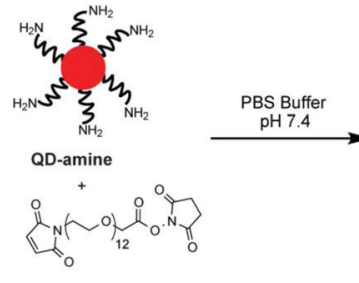

Bifunctional Linker

B

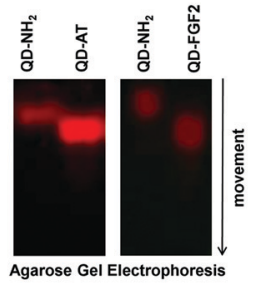

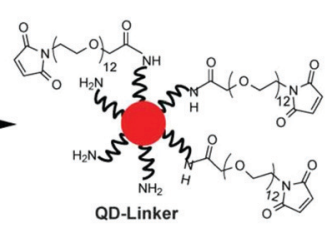
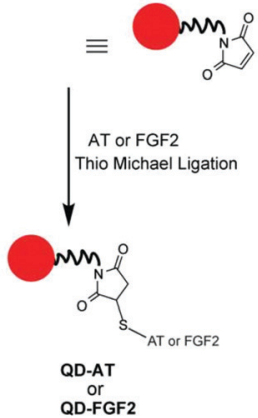

Fig. 2 Quantum dot (QD) bioconjugation of antithrombin (AT) or fibroblast growth factor2 (FGF2). (A) QD-NH2 was first modified with a bifunctional tether to attach a Michael acceptor (maleimide) and then reacted with the protein in the presence of Cys to form disulphide bridged QD-AT or QD-FGF2 probes. (B) The synthesis of the probes was confirmed through $1 \%$ agarose gel electrophoresis, which showed faster moving labelled complex than the unlabelled QDs.

changes in sequence composition in vivo, which could help pinpoint strategies for addressing novel therapeutics.

Here, we report the construction of a quantum dot (QD)-based fluorescent probe that reveals the spatio-temporal topography of a rare HS motif. The QD-based probes are non-radioactive, photostable, highly sensitive (10-100-fold more than Alexa Fluor dyes), compatible with both optical and electron microscopy, and very useful for cell and in vivo imaging. ${ }^{17,22}$ We show that this readilyapplicable QD-based technology can dramatically enhance the precision of localization of rare motifs present in HSPGs on cell surfaces.

We synthesized two QD-based probes to visualize, compare and quantify the topography of rare and common HS motifs (Fig. 2). The first probe targeted the specific 3-O-sulfated pentasaccharide motif, which binds human plasma AT with high affinity, whereas an analogous QD-conjugated FGF2 probe assessed the common tetrasaccharide sequences of HS. Because neither AT nor FGF2 possess a free cysteine, we utilized a cysteine (or TCEP) reduction methodology to release - $\mathrm{SH}$ groups for bioconjugation with QDs (see Fig. S1, ESI $\dagger$ for mechanism), thereby ascertaining minimal alteration of HS recognition. In fact, fluorescence titrations with the high-affinity heparin pentasaccharide (H5) showed a $K_{\mathrm{D}}$ of $38 \mathrm{nM}$ for the QD-AT:H5 complex, which was similar to that of the AT:H5 complex ( $10 \mathrm{nM})$ under comparable conditions at $\mathrm{pH} 7.4$, I 0.85 and $25{ }^{\circ} \mathrm{C}$ (Fig. S3, ESI $\dagger$ ). ${ }^{23}$ We also measured a stoichiometry of nearly two AT molecules per every QD molecule (Fig. S4, ESI $\dagger$ ), which explains the significant difference in molecular size between native and conjugated QD species (Fig. 2B).

The specificity of the QD-AT probe was first assessed in wild-type Chinese hamster ovary (CHO-K1) cells that do not express 3-OST-1 enzyme, which results in cell surface HSPGs that are devoid of 3-OS

motifs. No QD-AT fluorescence $\left(\lambda_{\mathrm{EM}}=655 \mathrm{~nm}\right)$ was observed for these cells (Fig. 3A). In contrast, treatment of the CHO-K1 cells

A

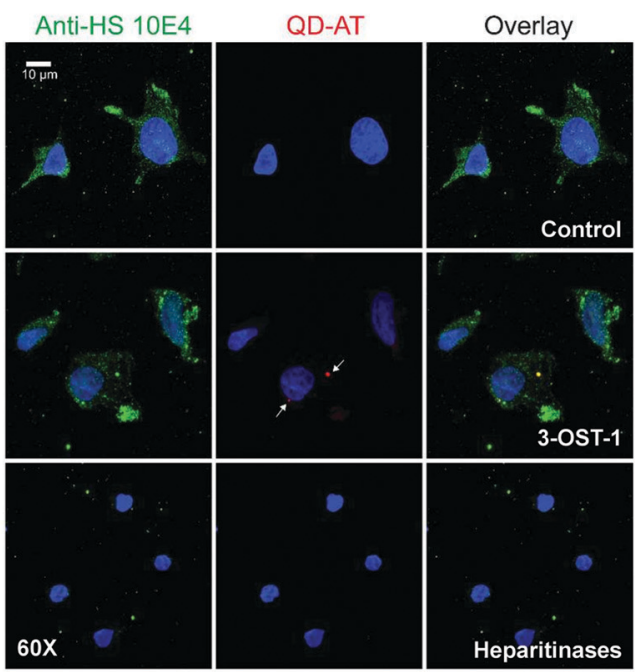

B
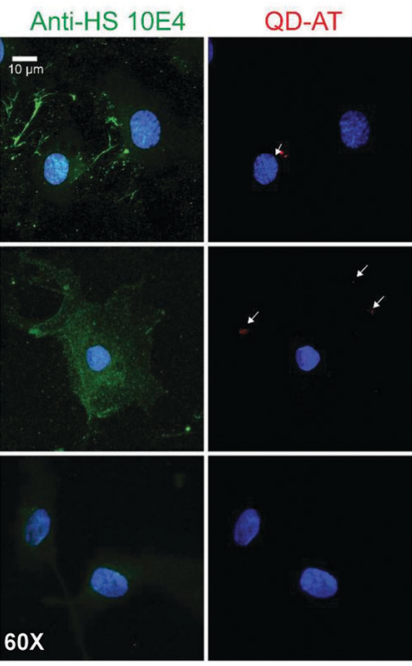

Overlay
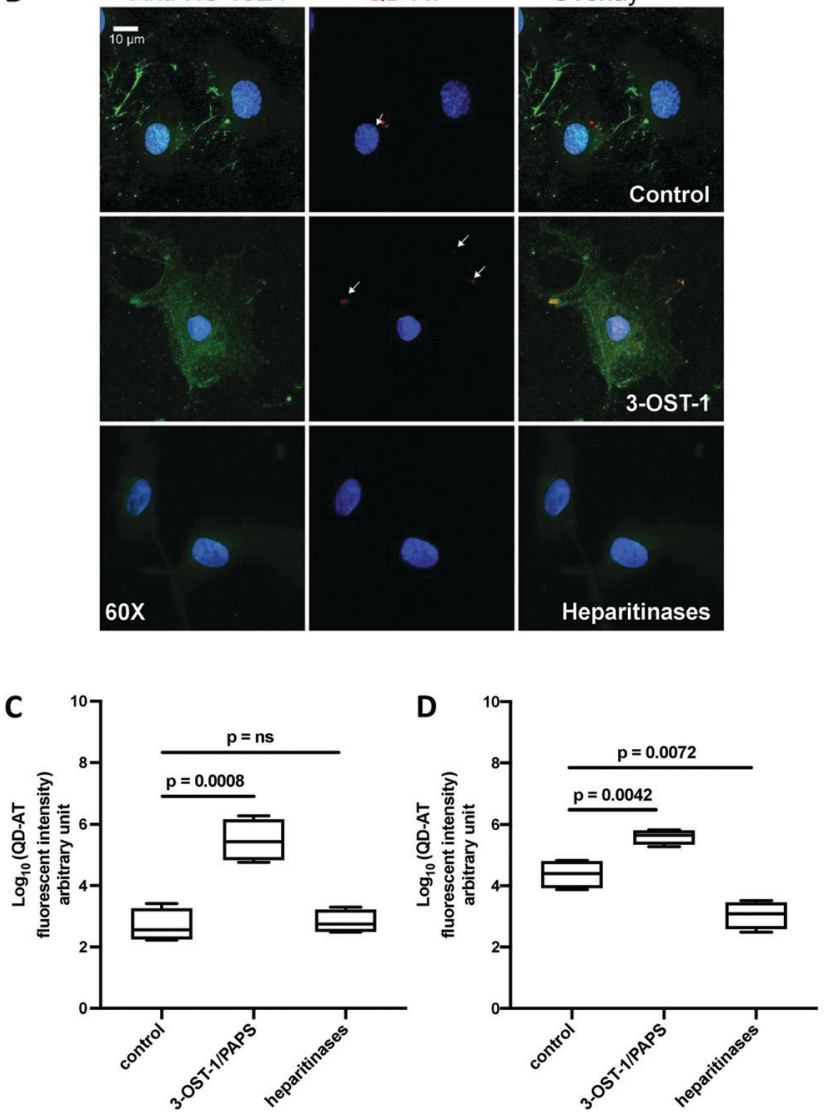

Fig. 3 Fluorescence imaging of the rare 3-O-sulfated HS motifs $\left(\lambda_{E M}=\right.$ $655 \mathrm{~nm}$ for QD-AT (red); $\lambda_{E M}=519 \mathrm{~nm}$ for anti-HS (10E4) antibody (green), and $\lambda_{\mathrm{EM}}=461 \mathrm{~nm}$ for DAPI (blue)). (A) CHO-K1 cells lacking 3-OST-1 expression (top); with 3-OST-1 and PAPS (middle); and cells with 3-OST-1 and PAPS, followed by heparitinases (bottom). Arrows mark the QD-AT probe on $\mathrm{CHO}-\mathrm{K} 1$ cell surface. Scale bar $=10 \mu \mathrm{m}$. (B) Corresponding experiments using bovine lung microvasculature cells (BLMVECS). (C) and (D) Pooled intensities quantified from panels A and B, respectively $(N=4)$. Statistical significance using student's $t$ test. 
with 3-OST-1 enzyme in the presence of the sulfate donor PAPS (3'-phosphoadenosine- $5^{\prime}$-phosphosulfate), which is known to generate 3-OS motifs on cell surface HSPGs, ${ }^{24,25}$ resulted in $\sim 614$-fold increase in the QD-AT fluorescence ( $p=0.0008$ ) (Fig. 3C).

Interestingly, the new motifs formed by 3-OST1/PAPS were bundled at specific loci on the CHO-K1 cells. Recent results show that 3-OST-1 recognizes substrate sequences very selectively, ${ }^{3}$ which is one of the major causes behind the rarity of this 3-OS structural motif. Further, the QD-AT-labelled HS subpopulation also overlapped with $10 \mathrm{E} 4$, an anti-HS epitope antibody that does not recognize any other GAGs, supporting the location of 3-OS motifs within HS chains. Finally, treatment of cells with a cocktail of heparitinases (Hep I, II and III), which degrade HS, resulted in a precipitous loss of the QD-AT signal (Fig. 3A).

Following validation in the 3-OST-1 lacking cell line (CHO-K1), we studied wild-type bovine lung microvasculature endothelial cells (BLMVECs). We observed occasional labelling of BLMVECs (Fig. 3B) with $<1 \%$ 3-OS motifs present on these cells, as documented in the literature. ${ }^{11}$ A 17 -fold rise in 3-OS labelling was observed with 3-OST-1 modification $(p=0.0042)$ (Fig. 3D). Once again, the newly introduced 3-OS motifs were localized in clusters on BLMVECs. Finally, the removal of HS chains from cell surface HSPG with heparitinase treatment ablated QD-AT fluorescence signals by $\sim 22$-fold $(p=0.0072$ ). This deduction was also supported by a similar reduction in signal from anti-HS antibody (10E4) (see Fig. S5, ESI $\dagger$ ).

Next, we used the QD-FGF2 probe to visualize the common and ubiquitous tetrasaccharide motif on CHO-K1 cells and BLMVECs (Fig. S6, ESI $\dagger$ ). Both cell types, when treated with QD-FGF2 probe, revealed a ubiquitous expression of the common tetrasaccharide motif all over the cell surface. As expected, the 3-OST-1/PAPS introduction hardly changed the QD-FGF2 signal suggesting that the 3-OS groups do not impact FGF2 recognition (Fig. S6, ESI $\dagger$ ). In contrast, heparitinase cocktail treatment ablated probe fluorescence for both cell types. Thus, the QD-FGF2 probe demonstrates a completely distinct ability to visualize HS motifs from the QD-AT probe.

The ability to visualize and quantify rare 3-OS motifs on cell surfaces led to the question whether the QD-AT probe would help visualize in vivo temporal changes, if any, of this rare population of HS motifs. We selected two C57BL/6 mouse groups, a two-weeks and a six-months old group $(N=3$ each), which would correspond to $\sim 2-6 \S$ and $\sim 20-69$ human years, $\S$ respectively. ${ }^{26}$ We sought to visualize changes arising from aging, if any, in the distribution and abundance of 3-OS motifs on the aorta and vasa vasorum, which deliver nutrients to the walls of blood vessels. ${ }^{27}$ The aortas of each animal groups were surgically removed and divided into two treatment cohorts - QDAT treatment and heparitinase followed by QD-AT treatment (see ESI $\dagger$ ). Fig. 4 shows representative results for the two groups and four cohorts. The six-months old group showed a 79-fold less fluorescence of the QD-AT probe in comparison to the two-weeks old group ( $p<0.0001)$. When treated with the heparitinase cocktail, a dramatic reduction in fluorescence intensities for both groups was observed, as expected. The loss in QD-AT fluorescence highlights the significant difference in the levels
A

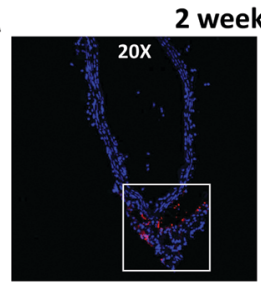

2 weeks aorta
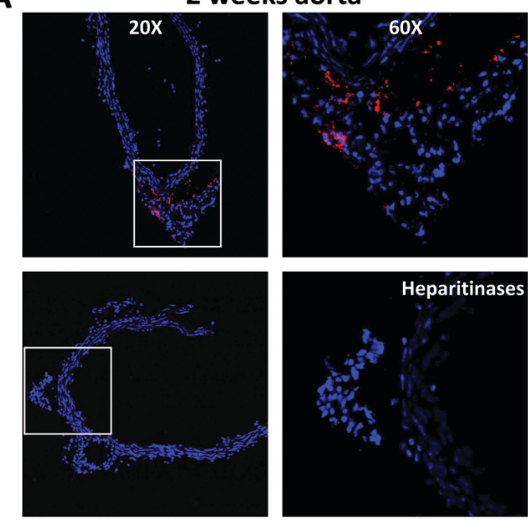

B

6 months aorta
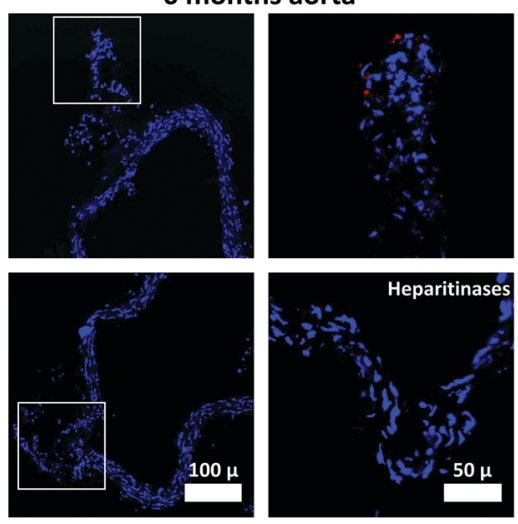

C

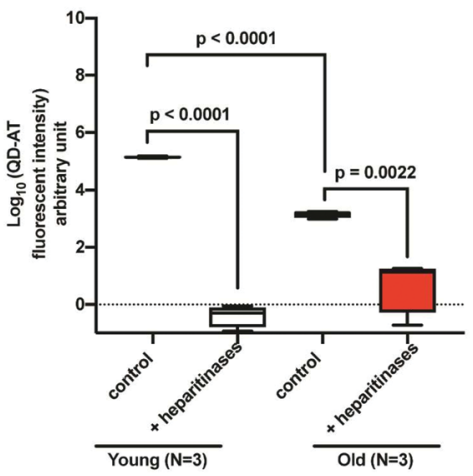

Fig. 4 C57BL/6 mouse aorta fluorescence imaging of the rare 3-OS HS motifs $\left(\lambda_{E M}=655 \mathrm{~nm}\right.$ for QD-AT (red) and $\lambda_{E M}=461 \mathrm{~nm}$ for DAPI (blue)). (A) The aorta of young animals ( $<2$ weeks) were compared with those from (B) older animals ( $>6$ months). (C) Intensities derived from a set of three animals $(N=3)$ and four different $20 \times$ cryo-section images. Statistical significance was calculated using student's $t$ test. Scale bar $=100 \mu \mathrm{m}$ for $20 x ; 50 \mu \mathrm{m}$ for $60 x$

of 3-OS motifs present on HSPGs lining the aortic and vasa vasorum endothelium as a function of age.

The high fidelity of QD-AT fluorescence quantitation also pointed to that possible difference in level of heparitinase action on the ECM of a formative young aorta versus the older aorta. Whereas heparitinase essentially eliminated QD-AT fluorescence in the young animal, some 3-OS motifs appeared to persist for the older aorta. It is possible that the extracellular matrix (ECM) architecture of the young and old aortic tissue differ. In fact, 
ECM stiffens with age ${ }^{28,29}$ which may impede enzymatic action of heparitinases.

Overall, we have demonstrated that using simple chemistry and QD-based imaging technology, it is possible to visualize the location as well as quantify the levels of rare 3-OS motifs of HS at cell surfaces. Our work elegantly shows that the technique is specific and robust for evaluating samples from live animals.

Our work also highlights two highly interesting biological results that will require further studies. (1) The 3-OS motifs were localized in distinct clusters on both CHO-K1 cells as well as BLMVECs. Although noted earlier, ${ }^{21}$ it would be important to assess its generality across a range of cells, especially from different organs. This observation could have major bearing on the selectivity, regulation and outcome of HS biosynthesis. ${ }^{1,3,13}$ (2) The 3-OS motifs were quantitatively down regulated in aged animals as compared to younger animals. It is well-known that the incidences of thrombosis and coronary arterial disease increase with aging. ${ }^{30,31}$ The underlying causes for these are many; ${ }^{31,32}$ yet, this study presents a new possibility. The reduction in AT-binding 3-OS motifs may reduce the anti-coagulation potential of the cell surface, thereby contributing to pro-thrombotic risk. Although much experimentation would be needed to test this hypothesis, our work demonstrates for the first time that the QD-based probes should be extremely useful in identifying dynamic changes in HS motifs arising from aging. This could have significant impact on diseases associated with 3-OS motifs such as thrombosis and neurodegeneration. ${ }^{4,9,33}$

Finally, the QD-based technology could visualize majority of rare HS motifs for which proteins are known to exhibit high selectivity, e.g., heparin cofactor II. ${ }^{34}$ Considering that a large number of proteins could bind HS selectively, ${ }^{35}$ QD-based probes should find use in elucidating the spatio-temporal dynamics of distinct HS motifs in vivo.

\section{Conflicts of interest}

There are no conflicts to declare.

\section{Notes and references}

\$ Residues include $\mathrm{N}$-sulfoglucosamine (GlcNS), $\mathrm{N}$-acetylglucosamine (GlcNAc), glucuronic acid (GlcA) and iduronic acid (IdoA) that may be sulfated at 2-, 3-, or 6-positions.

$\S$ Mice and humans have different rates of changes in different metabolic processes, which have been used to calculate an estimate of variation in correspondence between mouse and human years.

1 J. R. Bishop, M. Schuksz and J. D. Esko, Nature, 2007, 446, 1030-1037.

2 A. R. Rezaie and H. Giri, J. Thromb. Haemostasis, 2020, DOI: 10.1111/ jth.15052.

3 N. V. Sankaranarayanan, Y. Bi, B. Kuberan and U. R. Desai, Comput. Struct. Biotechnol. J., 2020, 18, 933-941.

4 U. R. Desai, Med. Res. Rev., 2004, 24, 151-181.

5 C. Lanzi and G. Cassinelli, Biochem. Pharmacol., 2020, 178, 114084, DOI: $10.1016 /$ j.bcp.2020.114084.
6 V. H. Pomin, Biochimie, 2016, 127, 214-216.

7 R. Goetz and M. Mohammadi, Nat. Rev. Mol. Cell Biol., 2013, 14, 166-180.

8 J. Schlessinger, A. N. Plotnikov, O. A. Ibrahimi, A. V. Eliseenkova, B. K. Yeh, A. Yayon, R. J. Linhardt and M. Mohammadi, Mol. Cell, 2000, 6, 743-750.

9 B. E. Thacker, D. Xu, R. Lawrence and J. D. Esko, Matrix Biol., 2014, $35,60-72$.

10 A. I. de Agostini, J.-C. Dong, C. de Vantéry Arrighi, M.-A. Ramus, I. Dentand-Quadri, S. Thalmann, P. Ventura, V. Ibecheole, F. Monge, A.-M. Fischer, S. H. Mohammadi, N. W. Shworak, L. Zhang, Z. Zhang and R. J. Linhardt, J. Biol. Chem., 2008, 283, 28115-28124.

11 J. A. Marcum, D. H. Atha, L. M. Fritze, P. Nawroth, D. Stern and R. D. Rosenberg, J. Biol. Chem., 1986, 261, 7507-7517.

12 G. Pejler, G. Bäckström, U. Lindahl, M. Paulsson, M. Dziadek, S. Fujiwara and R. Timpl, J. Biol. Chem., 1987, 262, 5036-5043.

13 J.-P. Li and M. Kusche-Gulberg, Int. Rev. Cell Mol. Biol., 2016, 325 , 215-273.

14 J. Kreuger and L. Kjellén, J. Histochem. Cytochem., 2012, 60, 898-907.

15 Z. Zou, Z. L. Luo, X. Xu, S. Yang, Z. H. Qing, J. W. Liu and R. Yang, TrAC, Trends Anal. Chem., 2020, 125, 115811.

16 B. N. G. Giepmans, S. R. Adams, M. H. Ellisman and R. Y. Tsien, Science, 2006, 312, 217-224.

17 X. Michalet, F. F. Pinaud, L. A. Bentolila, J. M. Tsay, S. Doose, J. J. Li, G. Sundaresan, A. M. Wu, S. S. Gambhir and S. Weiss, Science, 2005, 307, 538-544.

18 Y. Suzuki, C. N. Roy, W. Promjunyakul, H. Hatakeyama, K. Gonda, J. Imamura, B. Vasudevanpillai, N. Ohuchi, M. Kanzaki, H. Higuchi and M. Kaku, Mol. Cell. Biol., 2013, 33, 3036-3049.

19 M. J. Oh, J. Akhtar, P. Desai and D. Shukla, Biochem. Biophys. Res. Commun., 2010, 391, 176-181.

20 N. C. Smits, S. Kurup, A. L. Rops, G. B. ten Dam, L. F. Massuger, T. Hafmans, J. E. Turnbull, D. Spillmann, J. P. Li, S. J. Kennel, J. S. Wall, N. W. Shworak, P. N. Dekhuijzen, J. van der Vlag and T. H. van Kuppevelt, J. Biol. Chem., 2010, 285, 41143-41151.

21 A. I. de Agostini, S. C. Watkins, H. S. Slayter, H. Youssoufian and R. D. Rosenberg, J. Cell Biol., 1990, 111, 1293-1304.

22 U. Resch-Genger, M. Grabolle, S. Cavaliere-Jaricot, R. Nitschke and T. Nann, Nat. Methods, 2008, 5, 763-775.

23 U. R. Desai, M. Petitou, I. Björk and S. T. Olson, J. Biol. Chem., 1998, 273, 7478-7487.

24 L. Zhang, D. L. Beeler, R. Lawrence, M. Lech, J. Liu, J. C. Davis, Z. Shriver, R. Sasisekharan and R. D. Rosenberg, J. Biol. Chem., 2001, 276, 42311-42321.

25 B. Kuberan, D. L. Beeler, M. Lech, Z. L. Wu and R. D. Rosenberg, J. Biol. Chem., 2003, 278, 52613-52621.

26 S. Dutta and P. Sengupta, Life Sci., 2016, 152, 244-248.

27 J. K. Williams and D. D. Heistad, Trends Cardiovasc. Med., 1996, 6, 53-57.

28 C. M. Kelleher, S. E. McLean and R. P. Mecham, Curr. Top. Dev. Biol., 2004, 62, 153-188.

29 J. C. Kohn, M. C. Lampi and C. A. Reinhart-King, Front. Genet., 2015, 6, 112.

30 E. J. Favaloro, M. Franchini and G. Lippi, Semin. Thromb. Hemostasis, 2014, 40, 621-633.

31 C. Shao, J. Wang, J. Tian and Y. Tang, Adv. Exp. Med. Biol., 2020, 1177, 1-36.

32 K. Murata, J. Mol. Cell. Cardiol., 1981, 13, 281-292.

33 J. Zhao, Y. Zhu, X. Song, Y. Xiao, G. Su, X. Liu, Z. Wang, Y. Xu, J. Liu, D. Eliezer, T. F. Ramlall, G. Lippens, J. Gibson, F. Zhang, R. J. Linhardt, L. Wang and C. Wang, Angew. Chem., Int. Ed., 2020, 59, 1818-1827.

34 N. V. Sankarayanarayanan, T. R. Strebel, R. S. Boothello, K. Sheerin, A. Raghuraman, F. Sallas, P. D. Mosier, N. D. Watermeyer, S. Oscarson and U. R. Desai, Angew. Chem., Int. Ed., 2017, 56, 2312-2317.

35 N. V. Sankarayanarayanan, B. Nagarajan and U. R. Desai, Curr. Opin. Struct. Biol., 2018, 50, 91-100. 